\title{
Tracking post-infectious fatigue in clinic using routine Lab tests
}

\author{
Jeanna M. Harvey ${ }^{1}$, Gordon Broderick ${ }^{1,2,3^{*}}$, Alanna Bowie ${ }^{3}$, Zachary M. Barnes ${ }^{1}$, Ben Z. Katz ${ }^{4}$, \\ Maurice R. G. O'Gorman”, Suzanne D. Vernon ${ }^{6}$, Mary Ann Fletcher ${ }^{2}$, Nancy G. Klimas ${ }^{2}$ and Renee Taylor ${ }^{7}$
}

\begin{abstract}
Background: While biomarkers for chronic fatigue syndrome (CFS) are beginning to emerge they typically require a highly specialized clinical laboratory. We hypothesized that subsets of commonly measured laboratory markers used in combination could support the diagnosis of post-infectious CFS (PI-CFS) in adolescents following infectious mononucleosis (IM) and help determine who might develop persistence of symptoms.
\end{abstract}

Methods: Routine clinical laboratory markers were collected prospectively in 301 mono-spot positive adolescents, $4 \%$ of whom developed CFS ( $n=13)$. At 6, 12, and 24 months post-diagnosis with IM, 59 standard tests were performed including metabolic profiling, liver enzyme panel, hormone profiles, complete blood count (CBC), differential white blood count (WBC), salivary cortisol, and urinalysis. Classification models separating PI-CFS from controls were constructed at each time point using stepwise subset selection.

Results: Lower ACTH levels at 6 months post-IM diagnosis were highly predictive of CFS (AUC $p=0.02$ ). ACTH levels in CFS overlapped with healthy controls at 12 months, but again showed a trend towards a deficiency at 24 months. Conversely, estradiol levels depart significantly from normal at 12 months only to recover at 24 months (AUC $p=0.02$ ). Finally, relative neutrophil count showed a significant departure from normal at 24 months in CFS (AUC $p=0.01$ ). Expression of these markers evolved differently over time between groups.

Conclusions: Preliminary results suggest that serial assessment of stress and sex hormones as well as the relative proportion of innate immune cells measured using standard clinical laboratory tests may support the diagnosis of PI-CFS in adolescents with IM.

Keywords: Blood glucose, ACTH, Estradiol, Neutrophil count, Free thyroxin, Salivary cortisol, Classification models, Infectious mononucleosis, EBV, Chronic fatigue

\section{Background}

Chronic Fatigue Syndrome (CFS) is a complex, multisymptom illness involving persistent fatigue, musculoskeletal symptoms, as well as cognitive impairment [1]. Studies show that CFS affects between 1 and 4 million individuals $[2,3]$ and costs an estimated $\$ 35$ billion per year in health care and lost productivity $[4,5]$. CFS affects roughly $0.2 \%$ of the general adolescent population [6] and is a major cause of educational disruption [7-10]. While viral serology is not necessary for diagnosis, CFS follows infection in at least a subset

\footnotetext{
* Correspondence: gbroderick@nova.edu

'Department of Medicine, University of Miami, Miami, FL, USA

${ }^{2}$ Institute for Neuro Immune Medicine, Nova Southeastern University,

University Park Plaza, 3440 South University, Fort Lauderdale 33328, FL, USA

Full list of author information is available at the end of the article
}

of cases $[1,11]$. In adults, symptom onset coincided with apparent infection in up to $72 \%$ of cases; [12] in adolescents, infectious mononucleosis (IM) is the most commonly reported antecedent infection [9, 13-15]. The reported incidence of CFS following IM is 9-12 \% [16-19]. Females appear more susceptible than males $[10,16,20$, 21]. Consistent with this, our group reported previously that 13,7 and $4 \%$ of adolescents met criteria for CFS at 6 , 12 and 24 months respectively post-IM, and at 6 months post-IM, $90 \%$ of the subjects with CFS were female, while at 12 and 24 months, $100 \%$ were female [22].

Diagnosis of CFS currently relies on exclusion of other medical or psychological causes [1, 23, 24]. Currently there are no validated diagnostic test, although Klimas et al. [25] have reviewed some candidate biomarkers. 
Confirming many but not all past reports, Brenu et al. [26] and Fletcher et al. [27] found natural killer (NK) cell cytotoxicity to be low. Fletcher et al. [28] also found evidence that Neuropeptide Y, an immunologically active neurotransmitter, was elevated in subjects with CFS and correlated with symptom severity. Mild hypocortisolism with attenuated diurnal variation has also been reported in some patients with CFS [29]. In addition to the early identification of potential individual candidate markers, studies using multiplex methods have reported broader surveys and more detailed analyses. For example, while Curriu et al. [30] found similar cell counts for T, B, and NK cell populations in general in healthy individuals and subjects with CFS $(n=22)$, the latter showed significant increases in the T-helper and regulatory $\mathrm{T}$ cell subsets. NK cells also showed higher levels of specific activation markers (e.g., CD69). Suggesting altered patterns of immune signaling, Broderick et al. [31] found that a subset of 5 cytokines used in combination supported an accuracy of better than $80 \%$ at a confidence level of 0.95 in separating subjects with post-IM CFS versus recovered controls.

In the present work we focused on common clinical laboratory assays measured longitudinally to explore whether a combination of such markers could act as a signature for CFS following IM. We reviewed commonly recorded laboratory data collected during a 2-year prospective study of 301 adolescents with mono-spot positive IM conducted by Katz et al. [22] in the greater Chicago area. In this study $4 \%$ of subjects met criteria for CFS per the Jason et al. revision [32] of the Fukuda criteria [1]. Fifty-nine standard blood, saliva, and urine tests, such as $\mathrm{CBC}$ with differential, metabolic profiling, and urinalysis, were performed at each time point. Linear classification models separating post-IM CFS from controls were constructed at each time point based on subsets of these markers selected iteratively. While the vast majority of these markers were uninformative, changes in $\mathrm{ACTH}$, estradiol, and relative neutrophil count across the 2-year period were discriminatory for post-infectious CFS (PI-CFS) with an accuracy ranging from 72 to $84 \%$.

\section{Methods}

\section{Cohort and clinical assessment}

A total of 301 adolescents, ages $12-18$, with a diagnosis of monospot positive acute IM were recruited on a referral basis from the greater Chicago area via school nurses, pediatric practices, and the Virology Laboratory of Children's Memorial Hospital (now the Ann \& Robert H Lurie Children's Hospital of Chicago) between September of 2004 and November of 2007. The presumption was that most cases of monospot-positive acute IM were caused by Epstein-Barr virus (EBV) infection. Of the 301 initially enrolled, 286 adolescents who were not fully recovered as well as those who recovered uneventfully (controls) successfully completed the telephone-based interview using the CFS Screening Questionnaire [33] and underwent evaluation at 6,12 , and 24 months postIM. Of these subjects, 13,7 , and $4 \%(n=13)$ respectively met the Jason et al. revision [32] of the Fukuda [1] case definition for CFS. The former also incorporates elements of the Canadian case definition reported in Carruthers et al. [23]. Ninety percent of subjects who met the CFS case definition at 6 months were female, and at 12 and 24 months, $100 \%$ of subjects who met the case definition were female. At 6, 12, and 24 months post-IM, blood, saliva, and urine samples were collected from cases and controls for laboratory analysis. Details of the full cohort are reported in Katz et al. [22]. In the present study we focus on the specific subset of $13 \mathrm{fe}$ male adolescents, representing $4 \%$ of the initial cohort of over 300 adolescents, who fit the case definition of CFS at 6, 12 and 24 months following diagnosis with IM. These adolescents were compared to $n=12$ female control subjects, case-matched on the basis of gender, age (+/- 1 year, with 1 exception) and Tanner breast and pubic hair stage ( 4 or 5 , with one exception), who recovered normally following the same infection. All subjects were menarchal. None of the patients were on significant, chronic medications. Five were on no medications. Four reported occasional inhalers for asthma, or Advil. Three were on birth control. One patient was on methylphenidate (Concerta), one had a history of clonidine use in the past and one reported 1 or 2 doses of Xanax per year because of fear of flying.

\section{Ethics statements}

All aspects of the study were approved by the Institutional Review Boards of Children's Memorial Research Center (now the Stanley Manne Research Institute) and the College of Applied Sciences of the University of Illinois at Chicago. Written informed consent was obtained from all participants age 18 years or older. For those subjects less than 18 years of age, written informed consent was obtained from their guardians, coupled with mandatory child assent. Secondary analysis of the biological samples and data was reviewed and approved by the Institutional Review Board of the University of Alberta.

\section{Laboratory measurements}

Morning fasting blood samples were collected into ethylene diamine tetra acetic acid (EDTA) anticoagulant tubes from cases and controls at 6, 12, and 24 months following the diagnosis of monospot positive, acute IM. Where appropriate plasma was separated within $2 \mathrm{~h}$ of collection and stored at $-80 \mathrm{oC}$ until assayed. A total of 59 standard laboratory tests were performed according 
to Good Clinical Laboratory Practice standards on blood and urine, including complete blood count with differential, metabolic profiling, erythrocyte sedimentation rate, liver enzymes, thyroid, adrenal, and sex hormone profiling, and urinalysis. Cortisol was isolated from passive drool with a solid phase extraction protocol using a Micromass Quattro Micro triple-quadrupole mass spectrometer equipped with a Z-spray ion source and a Waters 2795 Alliance HT HPLC system as described in Katz et al. [34] Summary statistics for the expression of these markers are shown in Additional file 1: Tables S1a-d.

\section{Statistical analyses}

To assess the significance of changes in the mean expression of individual markers occurring across each of these patient groups versus recovering controls, we used a standard parametric $t$-test after performing a logarithmic transformation. In conjunction with this, a nonparametric Wilcoxon rank-sum test was used to compare the difference in group-wise median expression for each marker at each time point. The significance of the effects for time and time $\mathrm{x}$ group interactions were assessed using a conventional 2-way ANOVA and verified further using a regression-based repeated measures analysis. These calculations were performed using the annovan as well as the fitrm and ranovatbl functions available in the MatLab Statistics Toolbox (The MathWorks, Inc., Natick, MA).

Individual markers were assessed using different laboratory assays. In conventional multiple testing problems this heteroscedasticity or non-uniform variance can be removed by rescaling. However, when the null hypothesis involves correlated markers as it does here, such a rescaling step is known to distort the result and methods to address this issue still constitute an active field of research [35]. Thus, raw null probability $p$-values are more appropriate in this situation determining the significance of group-wise changes. Nevertheless, we estimated false discovery rates (FDR) using both the more exploratory measure proposed by Storey [36] and the more conservative measure of Benjamini and Hochberg [37]. In the case of the Storey [36] FDR, we estimated the tuning parameter lambda $(\lambda)$ using both a bootstrap method and a polynomial regression. We found the latter to support more conservative estimates of FDR in this data set. FDR calculations were performed using the mafdr function available in the MatLab Statistics Toolbox (The MathWorks, Inc., Natick, MA).

In addition, we examined patterns of expression in multiple markers at the level of individual subjects to address the non-homogeneous nature of these groups. This was done by constructing linear discriminant classification models using a stepwise feature selection method to mitigate the effect of cross-correlation between markers [38]. Such regression models favor marker subsets that are minimally redundant [39]. Model terms were selected sequentially based on their respective partial-F test values. Markers with a null probability $\mathrm{p}($ partial $\mathrm{F})<0.05$ were selected for recruitment into the regression model while those currently in the model but showing a revised $\mathrm{p}($ partial $\mathrm{F})>0.10$ were removed. In the resulting model, an observed row $\times$ from the sample set is classified into group I rather than group $J$ if $0<B_{0}+x^{*} B$, where the coefficient vector $B$ and intercept vector $B_{0}$ are estimated from the data. All markers were normalized a priori using a conventional $\mathrm{z}$-score to a mean value of 0 and a variance of 1.0 with observations at 6 months serving as the reference datum. Classifiers were evaluated based on their overall error rate (incorrectly classified/total classified) in assigning subjects to their proper diagnostic group. Detection sensitivity and assignment specificity were then computed over a range of threshold values $B_{0}$ to produce a receiver-operator characteristic (ROC) curve [40]. These calculations were performed using the classify, classperf, rankfeatures and randfeatures functions available in the MatLab Statistics Toolbox and the MatLab Bioinformatics Toolbox (The MathWorks, Inc., Natick, MA) as well as with the SPSS Statistics Release 21.0 software package (IBM, New York, NY). For a further introduction and review of these statistical measures as they might apply more to clinical practice we refer the reader to $[41,42]$.

\section{Results}

The results of the parametric t-tests and nonparametric Wilcoxon rank-sum tests performed on all standard blood work, salivary tests, and urinalysis measures are presented in Additional file 1: Tables S1a-d. At 6 months, those with PI-CFS were found to have depressed glucose $(p=0.03$, ranksum $p=0.05)$ and depressed ACTH $(p=0.01$, ranksum $p=0.02)$ compared with controls. At 12 months, those with PI-CFS were found to have depressed estradiol compared with controls $(p=0.01$, ranksum $p=0.02)$, and at 24 months, those with PI-CFS had higher levels of neutrophils compared with controls $(p=0.02$, ranksum $p=0.01$ ). Although this did not meet statistical significance, we also found elevated free thyroxine (T4) at both $6(p=0.07)$ and 12 months $(p=0.08)$. In this groupwise comparison conducted separately at each time point none of the $p$-values obtained, whether by $t$ test or ranksum test, corresponded to a FDR of less than 0.20. This suggests that if values are considered independent from one visit to the next the probability of an incidental finding is high. However when analyzing the full time course using a 2-way ANOVA (Additional file 2: Table S2), we found a significant group effect for elevated free thyroxine 
(T4) $(p=0.01 ; \quad \mathrm{FDR}=0.01, \quad \mathrm{FDR} \quad \mathrm{BH}=0.26)$ and depressed morning salivary cortisol $(p=0.03$; FDR $=0.02$, FDR $\left.{ }_{\mathrm{BH}}=0.29\right)$ as well as significant time $(p=0.01$; $\mathrm{FDR}=0.02, \quad \mathrm{FDR} \mathrm{BH}=0.11$ ) and group effects for $\operatorname{ACTH~}\left(p=0.01 ;\right.$ FDR $=0.03$, FDR $\left.{ }_{\text {BH }}=0.26\right)$. All three of these markers, namely free thyroxine (T4), morning salivary cortisol and ACTH demonstrated group effects that satisfied a FDR $<0.05$ based on Storey [36] using a polynomial estimate for the tuning factor lambda $(\lambda)$. In none of the markers studied were we able to find a change in the slope of the trend from one subject group to the next (i.e., time $\times$ group effect). These results were largely confirmed when performing a complementary analysis using a regressionbased repeated measure model (Additional file 3: Tables S3a - d) with the exception of a few minor differences in the significance of the time effect only. Once again no significant time $\mathrm{x}$ group effects were identified.

When linear regression models were applied, one variable, unique at each time point, was selected as being the most suitable to distinguish between individuals recovering normally and those who progressed to PI-CFS. At 6, 12, and 24 months, ACTH, estradiol, and neutrophils, respectively, were highly discriminatory for PICFS. As shown in Table 1, at 6 months, the model based on depressed ACTH levels produced an accuracy of $72 \%$, a negative predictive value (NPV) of $73 \%$, positive predictive value (PPV) of $71 \%$, specificity of $67 \%$, and sensitivity of $77 \%$. In comparison, low glucose levels supported a slightly higher PPV (75\%) and specificity (75\%), but lower NPV (69\%) and sensitivity (69\%). Similarly at 12 months, the model based on depressed estradiol alone produced an accuracy of $76 \%$, a NPV of
$75 \%$, a PPV of $77 \%$, with a specificity of $75 \%$, and a sensitivity of $77 \%$. The classification accuracy increases as the sample time approaches 24 months. When applied to subject classification at 24 months, the model based on increased neutrophil count produced an accuracy of $84 \%$ corresponding to a NPV of $83 \%$, PPV of $85 \%$, specificity of $83 \%$, and sensitivity of $85 \%$.

Table 2 shows a summary of receiver operating characteristic (ROC) performance for classification of PI-CFS subjects at 6,12 , and 24 months post-diagnosis of IM using linear discriminant models with terms selected as optimal at each time point. Classification for the eventual onset of post-IM CFS at the 2-year time point based on ACTH levels measured at 6 months produced an area under the curve (AUC) of $0.77(p=0.02)$. In comparison, glucose measured at 6 months only supports an AUC value of $0.73(p=0.05)$. Estradiol levels measured at 12 months produced a classification for illness at 24 months with an AUC value of $0.78(p=0.02)$. Finally, illness at 24 months was predicted based on the relative level of neutrophils at that same time point with a highly significant value for the AUC of $0.83(p=0.01)$. The ROC curves produced based on measures taken at each time point are presented in Fig. 1 for the markers selected by stepwise regression, namely $\mathrm{ACTH}$, estradiol and relative neutrophil count. The evolution across the 2 -year period of all 4 differentially expressed markers is presented in Fig. 2.

\section{Discussion}

The diagnosis of CFS currently relies on the exclusion of other medical and psychiatric diseases. We sought to determine if standard laboratory analysis of blood, salivary, and urine markers used alone or in combination might

Table 1 Identification and performance of linear classification models. Classification of PI-CFS and healthy recovered control (RC) subjects at 6, 12 and 24 months post-diagnosis with IM using linear discriminant models with terms identified as optimal at each time point based on classical stepwise variable selection with the exception of classification based on glucose levels at 6 months which was specified based on differential expression

\begin{tabular}{|c|c|c|c|c|c|c|c|c|c|}
\hline Model & Assigned RC & Assigned PI-FS & Total & & Accuracy & NPV & PPV & Specificity & Sensitivity \\
\hline \multicolumn{10}{|l|}{6 months } \\
\hline \multirow[t]{2}{*}{ ACTH } & 8 & 4 & 12 & True RC & 0.72 & 0.73 & 0.71 & 0.67 & 0.77 \\
\hline & 3 & 10 & 13 & True PI-CFS & & & & & \\
\hline \multirow[t]{2}{*}{ Glucose } & 9 & 3 & 12 & True RC & 0.72 & 0.69 & 0.75 & 0.75 & 0.69 \\
\hline & 4 & 9 & 13 & True PI-CFS & & & & & \\
\hline \multicolumn{10}{|l|}{12 months } \\
\hline \multirow[t]{2}{*}{ Estradiol } & 9 & 3 & 12 & True RC & 0.76 & 0.75 & 0.77 & 0.75 & 0.77 \\
\hline & 3 & 10 & 13 & True PI-CFS & & & & & \\
\hline \multicolumn{10}{|l|}{24 months } \\
\hline \multirow[t]{2}{*}{ Neutrophils } & 10 & 2 & 12 & True RC & 0.84 & 0.83 & 0.85 & 0.83 & 0.85 \\
\hline & 2 & 11 & 13 & True PI-CFS & & & & & \\
\hline
\end{tabular}


Table 2 Summary of receiver operating characteristic (ROC) performance. Area under the classification curve (AUC) for assignment of PI-CFS subjects at 6, 12 and 24 months post-diagnosis with IM using a linear discriminant models with terms selected as optimal at each time point using s stepwise selection method. Classification performance based on glucose levels measured at 6 months is included for comparison and was specified on the basis of $t$ and ranksum tests

\begin{tabular}{llllll}
\hline & Area & Std. error ${ }^{a}$ & Asymptotic Sig. & & \multicolumn{2}{l}{ Asymptotic 95 \% Conf. interval } \\
\cline { 5 - 6 } Model & & & Lower bound & Upper bound \\
\hline ACTH (6 months) & 0.77 & 0.10 & 0.02 & 0.58 & 0.96 \\
Glucose (6 months) & 0.73 & 0.11 & 0.05 & 0.52 & 0.95 \\
Estradiol (12 months) & 0.78 & 0.09 & 0.02 & 0.60 & 0.97 \\
Neutrophil (24 months) & 0.83 & 0.09 & 0.64 & 1.00 \\
\hline
\end{tabular}

The test result variable(s): acth1 has at least one tie between the positive actual state group and the negative actual state group. Statistics may be biased ander the nonparametric assumption

${ }^{\mathrm{b}}$ Null hypothesis: true area $=0.5$

${ }^{c}$ Model specified on the basis of $t$ and ranksum tests, not as a result of stepwise selection

support the identification of individuals likely to develop PI-CFS. In the adolescent population studied we found significantly lower levels of glucose and ACTH 6 months following IM in individuals who 18 months later would continue to suffer with persistent symptoms. Glucose was not selected as a distinguishing variable using stepwise regression even though it displayed similar predictive power because of a strong overlap with similar changes in ACTH. Changes in glucose homeostasis during infection are context-dependent and poorly understood [43] with cytokines such as IL-6 reportedly promoting hypoglycemia during acute LPS-induced inflammation [44]. Interestingly relative expression of the latter emerged as a distinguishing feature for this illness group in our previous work [31]. In addition, significantly lower levels of estradiol and increased relative neutrophil count were also observed 12 and 24 months post-IM (Table 1). The accuracy of each individual marker in identifying individuals who develop PI-CFS at 24 months ranged between $72-84 \%$. The fact that different markers discriminated better at different times might highlight the dynamic nature of the immune and



Fig. 1 Performance of subject classification based on a minimal set of standard clinical assays. ROC curves for classification of subjects who went on to develop post-infectious chronic fatigue syndrome and subjects who recovered normally using a linear discriminant model selected as optimal based on data collected at 6,12 and 24-month time points using a stepwise selection method. Coefficients were tuned based on data collected at each time point only 

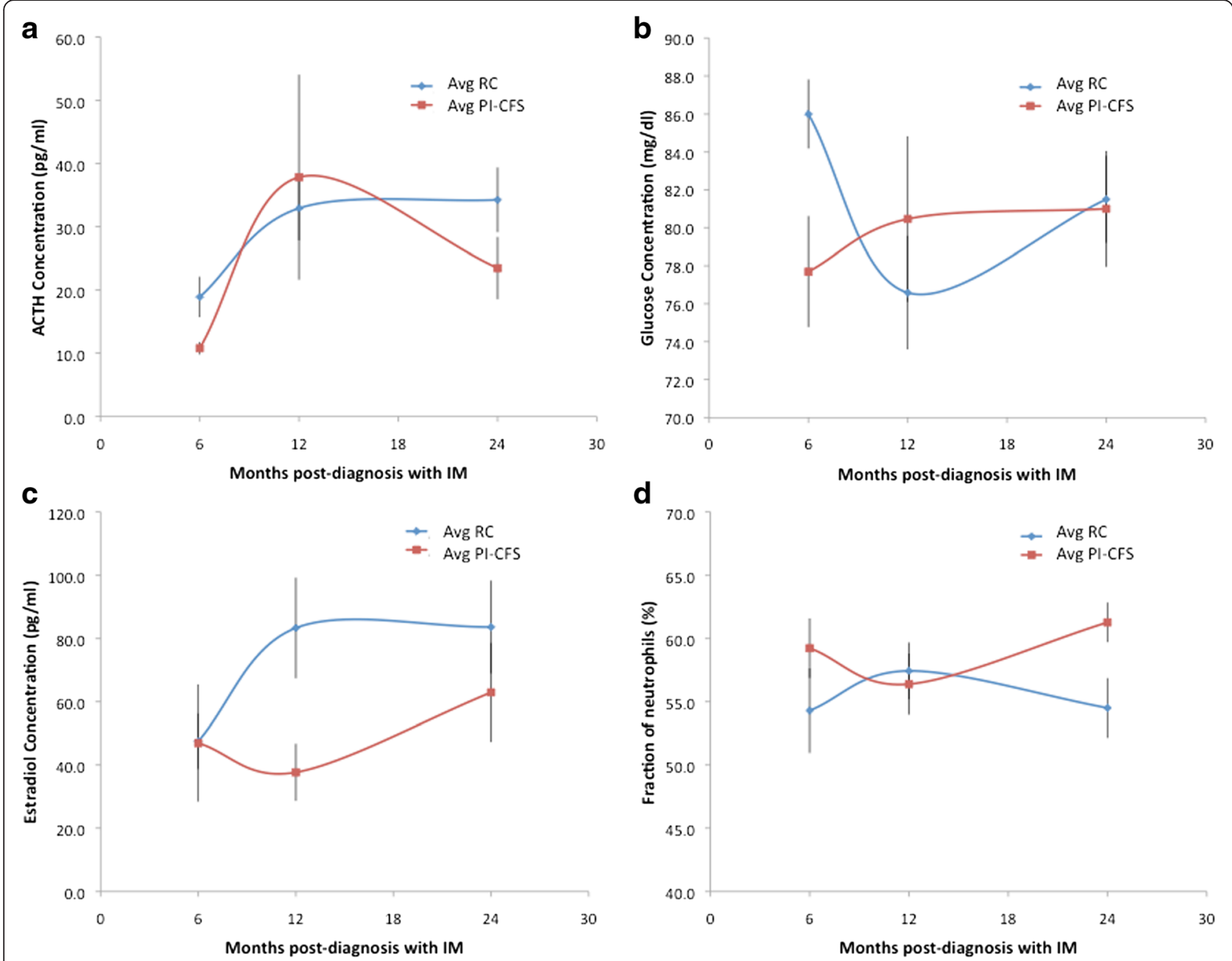

Fig. 2 Time course progression in expression of key discriminatory makers. Evolution of ACTH concentration (a), glucose concentration (b), estradiol concentration (c) and neutrophil count (d) in blood collected at 6, 12 and 24 months post-diagnosis with IM in subjects who went on to develop post-infectious chronic fatigue syndrome (PI-CFS; red square) and normally recovered control subjects (RC; blue triangle)

endocrine responses to this insult, which could be characterized as progressive. In several markers a persistent offset appears to be acquired quite early however the nonlinear nature of these changes is not easily captured by traditional linear analysis, e.g., the 2-way ANOVA employed herein. Because of this, single assessments will be of limited value while serial assessments within the first year may be much better suited for aiding in diagnosis of these subjects (e.g., serial estradiol measurements).

CFS is a complex constellation of symptoms [25]. Cannon et al. [45] found sex hormone driven changes in neutrophil count characteristic of CFS. The latter reported that while neutrophil count normally increases from the follicular to luteal phase in healthy women, it persists at luteal levels in CFS. Niblett et al. [46] also found that the neutrophil count and ratio of neutrophils to lymphocytes were both increased in CFS subjects. Discrepancies in neutrophil function have also been observed in some subjects with CFS with an overabundance of pre-apoptotic cells [47, 48]. Significant alterations in NK cell signaling and function have also been observed in some populations with CFS such as in our previous work in older women, where the elimination of target erythroleukemic K562 cells by NK cells was significantly impaired in CFS [27]. However Katz et al. [34] studying a similar population to that reported here found normal NK cell number and function in adolescents with CFS following IM. In addition to differences in the ages of the subjects between the two previously cited studies, there were also differences in methodology; the latter assessed cytotoxic function of NK cell isolates while the former studied NK cell function in a mixed lymphocyte population.

Involvement of sex-hormones in CFS is also consistent with our earlier work [49] and that of others, in which women were significantly more affected by this illness 
than men $[10,16,22]$. Our recent analysis using a theoretical model of regulatory physiology linking the hypothalamic-pituitary-adrenal (HPA) axis, the hypothalamic-pituitary-gonadal (HPG) and the peripheral immune system have shown that these interacting systems are especially prone in women to support chronically depressed cortisol levels and a persistent inflammatory signature consistent in some CFS populations [50].

Dysregulation of normal hypothalamic-pituitaryadrenal (HPA) axis function has been reported previously in patients with CFS [51]. Again, in our previous study from this population, we found no evidence of consistently depressed salivary cortisol in adolescents with CFS compared with recovered controls when individual time points were compared, although 3 of 9 subjects with CFS did have depressed morning cortisol (versus 1 of 9 controls). We concluded that depressed salivary cortisol may be an uncommon factor in CFS as even in those cases where it might play a role it did not persist despite persistence of symptoms [34]. In the current report, we found evidence of a group effect for depressed morning salivary cortisol as well as possibly other elements of hypothalamic-pituitary-thyroid (HPT) dysregulation with elevated free T4 at both $6(p=0.07)$ and 12 months $(p=0.08)$, which trended towards significance. These findings are also consistent with Fuite et al. [52] who found evidence of re-modeling in neuroendocrine and immune signaling in CFS. Specifically, results suggested a decreased cohesiveness in HPA axis response that coincided with a characteristic coexpression of immune markers with thyroid hormones in a larger group $(n=39)$ female CFS subjects. Changes in HPA axis feedback dynamics have also been reported recently by Aschbacher et al. [51] who describe an abnormal increase in negative feedback along the HPA axis in CFS. If examined individually, the observed decreases in these markers, while statistically significant, may escape biological significance. For example, estradiol levels from 10 to 260 are all normal for either the luteal or follicular phases of the menstrual cycle. Our study is an ad hoc analysis of prospectively collected data. If confirmed prospectively, depressed ACTH and possibly glucose might offer an objective way to confirm the diagnosis of CFS at 6 months following IM, while persistence of increased T4 and decreased morning salivary cortisol coupled with decreased estradiol at 12 months and increased neutrophil counts at 24 months might provide objective evidence for the persistence of CFS. Whether any of these findings would be helpful earlier than 6 months following IM is not known.

Limitations of this exploratory study include the small number of subjects $(n=13)$ and the relatively coarse sampling interval; nonetheless these were prospectively collected data from a study of PI-CFS with a welldefined trigger (IM). While these findings may not be applicable to the entire CFS population, a significant subset thereof reports onset coinciding with infectious illness. Though it is necessary validate these findings in a larger cohort, these initial results support the potential opportunity to identify individuals most at risk for developing CFS following IM and who might benefit from a more pro-active treatment. We purposely limited our assessment to common clinical markers in blood, saliva, and urine; while these readily available tests may not have the resolution to make a diagnosis, they may be sufficiently robust to support one. Indeed, additional means of confirmation using more focused laboratory assays as well as physiologic challenges to amplify these differences are being studied [53]. For example, in applying a maximal exercise challenge to this cohort our group found significantly higher oxygen consumption, work slope, and peak oxygen pulse in recovered subjects versus patients diagnosed with PI-CFS at 6 months after IM [54]. Other groups are reporting that such differences increase several fold in a follow-up challenge performed $24 \mathrm{~h}$ later [55] suggesting that a stress test may be much more revealing than a standard assessment. These limitations not withstanding, we found evidence that the onset of PI-CFS may be associated with the expression of specific patterns of markers in standard blood work. Whether these markers are etiologically related to CFS also remains to be determined and further studies should include at least one comparator illness common to this age group.

\section{Conclusions}

In a first exploratory analysis of a clinical data set we found that assessment of stress and sex hormones as well as the relative proportion of innate immune cells using standard clinical laboratory tests may help support the diagnosis of PI-CFS in adolescents with IM. Specifically, early course deviations in $\mathrm{ACTH}$ and possibly glucose along with subsequent differences in the levels of morning salivary cortisol, T4 and estradiol might provide objective evidence for the onset and persistence of CFS. As the relative deviations in these markers evolve over time, their contribution to accuracy and robustness of classification is greatly improved if they are recorded longitudinally.

\section{Additional files}

\footnotetext{
Additional file 1: Table S1a. Summary descriptive statistics of clinical assays. Mean and standard deviation () of the conventional complete metabolic profiling in blood at 6, 12 and 24 months following diagnosis with IM. Table $\mathbf{S 1 b}$. Summary descriptive statistics of clinical assays. Mean and standard deviation () of the conventional complete blood count (CBC) profiling in blood at 6, 12 and 24 months following diagnosis with IM. Table S1c. Summary descriptive statistics of clinical assays. Mean and
} 
standard deviation () of the conventional differential blood count profiling in blood at 6, 12 and 24 months following diagnosis with IM. Basophil count was not considered due to a large proportion of missing values. Table S1d. Summary descriptive statistics of clinical assays. Mean and standard deviation () of the conventional endocrine profiling in blood with urine specific gravity and pH at 6, 12 and 24 months following diagnosis with IM. (DOC $149 \mathrm{~kb}$ )

Additional file 2: Table S2a. Summary of 2-way ANOVA. Significance of time, group and time $x$ group effects of conventional metabolic profiling in blood across 6, 12 and 24 months following diagnosis with IM. False discovery rates (FDR) were based on Storey [36] using the bootstrap (boot) and the polynomial (poly) fit methods to estimate lambda, as well as on by Benjamini and Hochberg [37] (BH). Table S2b. Summary of 2-way ANOVA. Significance of time, group and time $x$ group effects for complete blood count (CBC) profiling in blood across 6, 12 and 24 months following diagnosis with IM. False discovery rates (FDR) were based on Storey [36] using the bootstrap (boot) and the polynomial (poly) fit methods to estimate lambda, as well as on by Benjamini and Hochberg [37] (BH). Table S2c. Summary of 2-way ANOVA. Significance of time, group and time $x$ group effects for conventional differential blood count profiling in blood across 6, 12 and 24 months following diagnosis with IM. False discovery rates (FDR) were based on Storey [36] using the bootstrap (boot) and the polynomial (poly) fit methods to estimate lambda, as well as on by Benjamini and Hochberg [37] (BH). Basophil count was not considered due to a large proportion of missing values. Table S2d. Summary of 2-way ANOVA. Significance of time, group and time $x$ group effects for standard endocrine profiling in blood with urine specific gravity and $\mathrm{pH}$ across 6,12 and 24 months following diagnosis with IM False discovery rates (FDR) were based on Storey [36] using the bootstrap (boot) and the polynomial (poly) fit methods to estimate lambda, as well as on by Benjamini and Hochberg [37] (BH). (DOC $150 \mathrm{~kb})$

Additional file 3: Table S3a,b,c and d. Supplemental tables containing summary statistics describing the significance and probability of false discovery for the effects of time and the time $\mathrm{x}$ group interaction for each of the clinical markers using a regression-based repeated measures model. (XLSX $48 \mathrm{~kb})$

\section{Abbreviations}

ACTH: adrenocorticotropic hormone; EBV: Epstein-Barr virus; IM: infectious mononucleosis; ME/CFS: myalgic encephalomyelitis/chronic fatigue syndrome.

\section{Competing interests}

The authors declare that they have no competing interests.

\section{Authors' contributions}

$\mathrm{JMH}, \mathrm{AB}, \mathrm{ZMB}$, and $\mathrm{GB}$ assembled the data and conducted all numerical analysis and drafted he initial manuscript. SDV, MO'G, MAF, BZK, RT, and NGK critically reviewed the manuscript. BZK and RT conceptualized, designed, and conducted the initial study. GB conceptualized, designed, and conducted this secondary analysis of the data. All authors read and approved the final manuscript.

\section{Acknowledgements}

This analysis was funded by the CFIDS Association of America grants to GB, BZK and NGK; all initial cohort recruitment and assessment were supported by US National Institutes of Health, R01 award HD043301-05 to PI RT, with secondary analysis also supported by R21AA016635 (PI MAF) and R01Al065723 (PI MAF); and R01AI065723 (PI MAF); the US Department of Veterans Affairs Merit Award to NGK.

\section{Author details}

${ }^{1}$ Department of Medicine, University of Miami, Miami, FL, USA. ${ }^{2}$ Institute for Neuro Immune Medicine, Nova Southeastern University, University Park Plaza, 3440 South University, Fort Lauderdale 33328, FL, USA. ${ }^{3}$ University of Alberta, Edmonton, AB, Canada. ${ }^{4}$ Ann \& Robert H Lurie Children's Hospital of Chicago, Chicago, IL, USA. ${ }^{5}$ Children's Hospital Los Angeles, Los Angeles, CA, USA. ${ }^{6}$ Solve ME/CFS Initiative, Charlotte, NC, USA. ${ }^{7}$ University of Illinois at Chicago, Chicago, IL, USA.
Received: 3 September 2014 Accepted: 21 April 2016 Published online: 26 April 2016

\section{References}

1. Fukuda K, Straus SE, Hickie I, Sharpe MC, Dobbins JG, Komaroff A. The chronic fatigue syndrome: a comprehensive approach to its definition and study. International Chronic Fatigue Syndrome Study Group. Ann Intern Med. 1994;121(12):953-9.

2. Chandler HK, Ciccone D, MacBride RJ, Natelson B. Medically unexplained illness in short- and long-term disability applicants: prevalence and cost of salary reimbursement. Disabil Rehabil. 2008;30(16):1185-91.

3. Reeves WC, Jones JF, Maloney E, Heim C, Hoaglin DC, Boneva RS, Morrissey M, Devlin R. Prevalence of chronic fatigue syndrome in metropolitan, urban, and rural Georgia. Popul Health Metr. 2007;5:5

4. Reynolds KJ, Vernon SD, Bouchery E, Reeves WC. The economic impact of chronic fatigue syndrome. Cost Eff Resour Alloc. 2004;2(1):4.

5. Jason LA, Benton MC, Valentine L, Johnson A, Torres-Harding S. The economic impact of ME/CFS: individual and societal costs. Dyn Med. 2008;7:6.

6. Jordan KM, Jason LA, Mears CJ, et al. Prevalence of pediatric chronic fatigue syndrome in a community-based sample. J of Chronic Fatigue Syndr. 2006;13:75-8.

7. Marshall GS, Gesser RM, Yamanishi K, Starr SE. Chronic fatigue in children: clinical features, Epstein-Barr virus and human herpesvirus 6 serology and long term follow-up. Pediatr Infect Dis J. 1991;10(4):287-90.

8. Richards J, Turk J, White S. Children and adolescents with Chronic Fatigue Syndrome in non-specialist settings: beliefs, functional impairment and psychiatric disturbance. Eur Child Adolesc Psychiatry. 2005;14(6):310-8.

9. Sankey A, Hill CM, Brown J, Quinn L, Fletcher A. A follow-up study of chronic fatigue syndrome in children and adolescents: symptom persistence and school absenteeism. Clin Child Psychol Psychiatry. 2006;11(1):126-38.

10. ter Wolbeek M, van Doornen LJ, Kavelaars A, Heijnen CJ. Severe fatigue in adolescents: a common phenomenon? Pediatrics. 2006;117(6):e1078-86.

11. Reeves WC, Lloyd A, Vernon SD, Klimas N, Jason LA, Bleijenberg G, Evengard B, White PD, Nisenbaum R, Unger ER. International Chronic Fatigue Syndrome Study Group. Identification of ambiguities in the 1994 chronic fatigue syndrome research case definition and recommendations for resolution. BMC Health Serv Res. 2003;3(1):25. Review.

12. Salit IE. Precipitating factors for the chronic fatigue syndrome. J Psychiatr Res. 1997;31(1):59-65.

13. Feder Jr HM, Dworkin PH, Orkin C. Outcome of 48 pediatric patients with chronic fatigue. A clinical experience. Arch Fam Med. 1994;3(12):1049-55. Review.

14. Krilov LR, Fisher M, Friedman SB, Reitman D, Mandel FS. Course and outcome of chronic fatigue in children and adolescents. Pediatrics. 1998;102(2 Pt 1):360-6.

15. Smith MS, Mitchell J, Corey L, Gold D, McCauley EA, Glover D, Tenover FC. Chronic fatigue in adolescents. Pediatrics. 1991;88(2):195-202.

16. Buchwald DS, Rea TD, Katon WJ, Russo JE, Ashley RL. Acute infectious mononucleosis: characteristics of patients who report failure to recover. Am J Med. 2000;109(7):531-7.

17. Hickie I, Davenport T, Wakefield D, Vollmer-Conna U, Cameron B, Vernon SD, Reeves WC, Lloyd A. Dubbo Infection Outcomes Study Group. Postinfective and chronic fatigue syndromes precipitated by viral and non-viral pathogens: prospective cohort study. BMJ. 2006;333(7568):575.

18. White PD, Thomas JM, Kangro HO, Bruce-Jones WD, Amess J, Crawford DH, Grover SA, Clare AW. Predictions and associations of fatigue syndromes and mood disorders that occur after infectious mononucleosis. Lancet. 2001; 358(9297):1946-54.

19. White PD, Thomas JM, Amess J, Crawford DH, Grover SA, Kangro HO, Clare AW. Incidence, risk and prognosis of acute and chronic fatigue syndromes and psychiatric disorders after glandular fever. Br J Psychiatry. 1998;173:475-81.

20. Rimes KA, Goodman R, Hotopf M, Wessely S, Meltzer H, Chalder T. Incidence, prognosis, and risk factors for fatigue and chronic fatigue syndrome in adolescents: a prospective community study. Pediatrics. 2007;119(3):e603-9.

21. Candy B, Chalder T, Cleare AJ, Peakman A, Skowera A, Wessely S, Weinman J, Zuckerman M, Hotopf M. Predictors of fatigue following the onset of infectious mononucleosis. Psychol Med. 2003;33(5):847-55.

22. Katz BZ, Shiraishi Y, Mears CJ, Binns HJ, Taylor R. Chronic fatigue syndrome after infectious mononucleosis in adolescents. Pediatrics. 2009;124(1):189-93.

23. Carruthers BM, Jain AK, De Meirleir KL, Peterson DL, Klimas NG, Lerner AM, Bested AC, Flor-Henry P, Joshi P, Powles ACP, Sherkey JA, van de Sande MI. Myalgic 
encephalomyelitis/chronic fatigue syndrome: clinical working case definition, diagnostic and treatments protocols. J Chron Fatigue Syndr. 2003;11:7-115.

24. Jason LA, Brown A, Evans M, Sunnquist M, Newton JL. Contrasting Chronic Fatigue Syndrome versus Myalgic Encephalomyelitis/Chronic Fatigue Syndrome. Fatigue. 2013;1(3):168-83.

25. Klimas NG, Broderick G, Fletcher MA. Biomarkers for chronic fatigue. Brain Behav Immun. 2012;26(8):1202-10. Review.

26. Brenu EW, van Driel ML, Staines DR, Ashton KJ, Ramos SB, Keane J, Klimas NG Marshall-Gradisnik SM. Immunological abnormalities as potential biomarkers in Chronic Fatigue Syndrome/Myalgic Encephalomyelitis. J Transl Med. 2011;9:81.

27. Fletcher MA, Zeng XR, Maher K, Levis S, Hurwitz B, Antoni M, Broderick G, Klimas NG. Biomarkers in chronic fatigue syndrome: evaluation of natural killer cell function and dipeptidyl peptidase IV/CD26. PLoS One. 2010;5(5):e10817.

28. Fletcher MA, Rosenthal M, Antoni M, Ironson G, Zeng XR, Barnes Z, Harvey JM, Hurwitz B, Levis S, Broderick G, Klimas NG. Plasma neuropeptide Y: a biomarker for symptom severity in chronic fatigue syndrome. Behav Brain Funct. 2010;6:76.

29. Papadopoulos AS, Cleare AJ. Hypothalamic-pituitary-adrenal axis dysfunction in chronic fatigue syndrome. Nat Rev Endocrinol. 2011;8(1):22-32.

30. Curriu M, Carrillo J, Massanella M, Rigau J, Alegre J, Puig J, Garcia-Quintana AM, Castro-Marrero J, Negredo E, Clotet B, Cabrera C, Blanco J. Screening NK-B- and T-cell phenotype and function in patients suffering from Chronic Fatigue Syndrome. J Transl Med. 2013;11:68.

31. Broderick G, Katz BZ, Fernandes H, Fletcher MA, Klimas N, Smith FA, O'Gorman MR, Vernon SD, Taylor R. Cytokine expression profiles of immune imbalance in post-mononucleosis chronic fatigue. J Transl Med. 2012;10:191.

32. Jason $L A$, Jordan $K$, Miike $T$, et al. A pediatric case definition for myalgic encephalomyelitis and chronic fatigue syndrome. J Chronic Fatigue Syndr. 2006:13:1-44

33. Jason LA, Richman JA, Rademaker AW, Jordan KM, Plioplys AV, Taylor RR, McCready W, Huang CF, Plioplys S. A community-based study of chronic fatigue syndrome. Arch Intern Med. 1999;159(18):2129-37.

34. Katz BZ, Zimmerman D, O'Gorman MRG, Mears CJ, Shiraishi Y, Taylor R Normal Salivary Cortisol and NK Cell Function in Adolescents With Chronic Fatigue Syndrome Following Infectious Mononucleosis. Arch Pediatr Infect Dis. 2013;1(5):211-6.

35. Sun W, McLain AC. Multiple Testing of Composite Null Hypotheses in Heteroscedastic Models. J Am Stat Assoc. 2012;107(498):673-87.

36. Storey JD. A direct approach to false discovery rates. J R Stat Soc. 2002;64(3):479-98.

37. Benjamini $Y$, Hochberg Y. Controlling the false discovery rate: a practical and powerful approach to multiple testing. J R Statist Soc B. 1995;57(1):289-300.

38. Rawlings JO. Collinearity Diagnostics. In: Barndorff-Nielsen OE, Bickel PJ, Cleveland WS, Dudley RM, editors. Applied Regression Analysis. Pacific Grove: Wadsworth and Brooks; 1988. p. 273-81.

39. Draper NR, Smith H. Stepwise Regression. In: Applied Regression Analysis. Hoboken, NJ: Wiley-Interscience; 1998. p. 307-12.

40. Greiner M, Pfeiffer D, Smith RD. Principles and practical application of the receiver-operating characteristic analysis for diagnostic tests. Prev Vet Med. 2000;45(1-2):23-41.

41. Zurakowski D, Johnson VM, Lee EY. Biostatistics in clinical decision making for cardiothoracic radiologists. J Thorac Imaging. 2013;28(6):368-75.

42. Langlotz CP. Fundamental measures of diagnostic examination performance: usefulness for clinical decision making and research. Radiology. 2003;228(1):3-9.

43. Nguyen AT, Mandard S, Dray C, Deckert V, Valet P, Besnard P, Drucker DJ, Lagrost L, Grober J. Lipopolysaccharides-mediated increase in glucose-stimulated insulin secretion: involvement of the GLP-1 pathway. Diabetes. 2014;63(2):471-82.

44. Tweedell A, Mulligan KX, Martel JE, Chueh FY, Santomango T, McGuinness OP. Metabolic response to endotoxin in vivo in the conscious mouse: role of interleukin-6. Metabolism. 2011;60:92-8.

45. Cannon JG, Angel JB, Abad LW, O'Grady J, Lundgren N, Fagioli L, Komaroff AL. Hormonal influences on stress-induced neutrophil mobilization in health and chronic fatigue syndrome. J Clin Immunol. 1998;18(4):291-8.

46. Niblett $\mathrm{SH}$, King KE, Dunstan RH, Clifton-Bligh $\mathrm{P}$, Hoskin LA, Roberts TK, Fulcher GR, McGregor NR, Dunsmore JC, Butt HL, Klineberg I, Rothkirch TB. Hematologic and urinary excretion anomalies in patients with chronic fatigue syndrome. Exp Biol Med (Maywood). 2007;232(8):1041-9.

47. Kennedy G, Spence V, Underwood C, Belch JJ. Increased neutrophil apoptosis in chronic fatigue syndrome. J Clin Pathol. 2004;57(8):891-3.
48. Brenu EW, Staines DR, Baskurt OK, Ashton KJ, Ramos SB, Christy RM, Marshall-Gradisnik SM. Immune and hemorheological changes in chronic fatigue syndrome. J Transl Med. 2010;8:1.

49. Smylie AL, Broderick G, Fernandes H, Razdan S, Barnes Z, Collado F, Sol C, Fletcher MA, Klimas N. A comparison of sex-specific immune signatures in Gulf War illness and chronic fatigue syndrome. BMC Immunol. 2013;14:29.

50. Craddock TJ, Fritsch P, Rice Jr MA, del Rosario RM, Miller DB, Fletcher MA, Klimas NG, Broderick G. A role for homeostatic drive in the perpetuation of complex chronic illness: Gulf War Illness and chronic fatigue syndrome. PLoS One. 2014;9(1):e84839.

51. Aschbacher K, Adam EK, Crofford LJ, Kemeny ME, Demitrack MA, Ben-Zvi A. Linking disease symptoms and subtypes with personalized systems based phenotypes: A proof of concept study. Brain Behav Immun. 2012;26(7):1047-56.

52. Fuite J, Vernon SD, Broderick G. Neuroendocrine and immune network re-modeling in chronic fatigue syndrome: an exploratory analysis. Genomics. 2008;92(6):393-9.

53. Maes M, Twisk FN, Johnson C. Myalgic Encephalomyelitis (ME), Chronic Fatigue Syndrome (CFS), and Chronic Fatigue (CF) are distinguished accurately: results of supervised learning techniques applied on clinical and inflammatory data. Psychiatry Res. 2012;200(2-3):754-60.

54. Katz BZ, Boas S, Shiraishi Y, Mears CJ, Taylor R. Exercise tolerance testing in a prospective cohort of adolescents with chronic fatigue syndrome and recovered controls following infectious mononucleosis. J Pediatr. 2010;157(3):468-72. 472.e1.

55. Snell CR, Stevens SR, Davenport TE, Van Ness JM. Discriminative validity of metabolic and workload measurements for identifying people with chronic fatigue syndrome. Phys Ther. 2013;93(11):1484-92.

\section{Submit your next manuscript to BioMed Central and we will help you at every step:}

- We accept pre-submission inquiries

- Our selector tool helps you to find the most relevant journal

- We provide round the clock customer support

- Convenient online submission

- Thorough peer review

- Inclusion in PubMed and all major indexing services

- Maximum visibility for your research

Submit your manuscript at www.biomedcentral.com/submit

) Biomed Central 